\title{
Clinical impact of digital and conventional PET control databases for semi-quantitative analysis of brain ${ }^{18} \mathrm{~F}-\mathrm{FDG}$ digital PET scans
}

\author{
Elise Mairal ${ }^{1}$, Matthieu Doyen ${ }^{1,2}$, Thérèse Rivasseau-Jonveaux ${ }^{3}$, Catherine Malaplate ${ }^{4}$, Eric Guedj ${ }^{5}$ \\ and Antoine Verger ${ }^{1,2^{*}}$ (1)
}

\begin{abstract}
Purpose: Digital PET cameras markedly improve sensitivity and spatial resolution of brain ${ }^{18} \mathrm{~F}$-FDG PET images compared to conventional cameras. Our study aimed to assess whether specific control databases are required to improve the diagnostic performance of these recent advances.

Methods: We retrospectively selected two groups of subjects, twenty-seven Alzheimer's Disease (AD) patients and twenty-two healthy control (HC) subjects. All subjects underwent a brain ${ }^{18} \mathrm{~F}$-FDG PET on a digital camera (Vereos, Philips ${ }^{\circledR}$ ). These two group (AD and HC) are compared, using a Semi-Quantitative Analysis (SQA), to two age and sex matched controls acquired with a digital PET/CT $\left(\right.$ Vereos, Philips ${ }^{\circledR}$ ) or a conventional PET/CT (Biograph 6, Siemens ${ }^{\circledR}$ ) camera, at group and individual levels. Moreover, individual visual interpretation of SPM T-maps was provided for the positive diagnosis of AD by 3 experienced raters.

Results: At group level, SQA using digital controls detected more marked hypometabolic areas in $\mathrm{AD}\left(+116 \mathrm{~cm}^{3}\right.$ at $p<0.001$ uncorrected for the voxel, corrected for the cluster) than SQA using conventional controls. At the individual level, the accuracy of SQA for discriminating AD using digital controls was higher than SQA using conventional controls ( $86 \%$ vs. $80 \%, p<0.01$, at $p<0.005$ uncorrected for the voxel, corrected for the cluster), with higher sensitivity ( $89 \%$ vs. $78 \%$ ) and similar specificity ( $82 \%$ vs. $82 \%$ ). These results were confirmed by visual analysis (accuracies of $84 \%$ and $82 \%$ for digital and conventional controls respectively, $p=0.01$ ).
\end{abstract}

Conclusion: There is an urgent need to establish specific digital PET control databases for SQA of brain ${ }^{18} \mathrm{~F}$-FDG PET images as such databases improve the accuracy of AD diagnosis.

\section{Introduction}

Digital PET cameras are composed of small digital silicon photomultipliers, which provide digital photon counting with a 1-to-1 crystal coupling, and replace the larger photomultiplier tubes of conventional PET cameras [1, 2]. These digital PET cameras thus provide improvements in detection sensitivity, spatial resolution and signal to noise

\footnotetext{
*Correspondence: a.verger@chru-nancy.fr

1 Department of Nuclear Medicine and Nancyclotep Imaging Platform, CHRU Nancy, Université de Lorraine, Rue du Morvan, 54500 Vandoeuvre-les-Nancy, France

Full list of author information is available at the end of the article
}

ratio and therefore in image quality compared to conventional PET cameras. This has been a particularly significant advance in brain ${ }^{18}$ F-FDG PET acquisitions [1, 2].

Brain ${ }^{18} \mathrm{~F}$-FDG PET is a useful tool for diagnosing neurodegenerative disorders. It is particularly useful in the diagnosis of Alzheimer's Disease (AD) [3], where a visual analysis of brain ${ }^{18}$ F-FDG PET images is initially performed to detect the typical $\mathrm{AD}$ hypometabolic pattern involving the posterior temporo-parietal association cortex $[4,5]$. Semi-quantitative analysis (SQA) has been proposed as an adjunct to this visual analysis since it increases confidence in the diagnostic conclusion 
drawn, particularly at earlier stages of the disease [6]. The importance of SQA has been further underpinned by its inclusion in the recommendations of the European Association of Nuclear Medicine and European Academy of Neurology [3].

The establishment of a well-documented reference database is a crucial step for performing SQA. A comparison to homogeneous control population databases is an important cornerstone to reduce false positives. Indeed, age and sex effects have been reported to influence metabolism distribution in brain ${ }^{18} \mathrm{~F}$-FDG PET images [7]. In addition, all currently implemented control databases in dedicated software for automated SQA are still based on acquisitions performed with conventional PET [8-13]. It is very likely that improvements in image quality provided by digital PET technology may also influence results of SQA, even though, to the best of our knowledge there is currently no data in the literature which evaluates the potential clinical impact of such an effect.

Our current study aims to assess whether there is a need to establish specific reference control databases which take into account the recent technical advances of SQA on brain ${ }^{18} \mathrm{~F}$-FDG PET images with respect to the evolution of digital PET.

\section{Materials and methods \\ Subjects}

We retrospectively selected two groups of subjects, a group of patients with $\mathrm{AD}$ and a group of healthy control subjects (HC). Both groups had undergone a brain ${ }^{18} \mathrm{~F}-$ FDG PET scan on a digital PET/CT (Vereos, Philips ${ }^{\circledR}$ ), at the CHRU of Nancy, France, between December 2017 and September 2019.

The AD patients selected fulfilled the NIA-AA 2018 criteria for AD [14]. They exhibited positive cerebrospinal fluid biomarkers with increased phosphorylated Tau protein and reduced beta-amyloid peptide levels measured in the same laboratory (Department of Biochemistry, Molecular Biology and Nutrition, CHRU Nancy, France) using standard cut-offs [15]. AD patients also underwent a routine neurocognitive assessment in the "memory clinic" of the university hospital of Nancy (France).

Healthy control subjects were age and sex matched with patients in the AD group and were also selected retrospectively. All healthy control subjects had undergone a brain ${ }^{18} \mathrm{~F}$-FDG PET scan for cognitive assessment, but had returned a normal scan by careful visual analysis (EM, AV) and a neuropsychological assessment which was not consistent with a neurodegenerative disorder: (i) normal neuropsychological tests, i.e. $M M S E \geq 27$, $\mathrm{FAB} \geq 15$ and no major depressive disorders and (ii) a clinical follow-up, of longer than 1 year, which showed a stabilisation and/or improvement of cognitive symptoms.

Our AD and $\mathrm{HC}$ groups were further compared to each other and to two control databases (a digital and a conventional database) derived from prospective studies. Individuals from these two control databases had undergone a brain ${ }^{18} \mathrm{~F}-\mathrm{FDG}$ PET/CT performed with a conventional camera between October 2009 to May 2012 $\left(n=19\right.$, Biograph 6 , Siemens ${ }^{\circledR}$, NCT02858167) or performed with a digital camera between December 2017 to June 2019 ( $n=20$, Vereos, Philips ${ }^{\circledR}$, NCT03345290) and were age and sex matched with our $\mathrm{AD}$ and $\mathrm{HC}$ groups. A flowchart summarising the constitution of the different control groups is shown in Fig. 1.

Informed consent was obtained for each participant included in the selected groups. This study was approved on January 16, 2020 by the local ethics committee (NCT04163276, Study ID Numbers: 2019PI238).

\section{Brain ${ }^{18}$ F-FDG PET}

The brain ${ }^{18}$ F-FDG PET scan was recorded over a 10 (for conventional camera) to 15 min (for digital camera) one bed acquisition, 45-50 min after injection of $4.5 \mathrm{MBq} /$ $\mathrm{kg}$ (conventional camera) or $2-3 \mathrm{MBq} / \mathrm{kg}$ (digital camera) of ${ }^{18}$ F-FDG. All subjects had fasted at least $6 \mathrm{~h}$ prior to receiving the injection and had blood glucose levels $<10 \mathrm{mmol} / \mathrm{L}$. All PET images were reconstructed with iterative OSEM methods, as performed in routine clinical practice, and corrected for scatter, random and attenuation with a CT scan. Reconstructed parameters included 4 iterations and 8 subsets, subsequently displayed in a $168 \times 168$ matrix with $2.7 \times 2.7 \times 2.7$ voxels for the conventional PET camera [7], and 3 iterations and 15 subsets, subsequently displayed in a $256 \times 256$ matrix with $1 \times 1 \times 1 \mathrm{~mm}^{3}$ voxels for the digital PET camera [2].

\section{Statistical parametric mapping}

The ${ }^{18}$ F-FDG PET brain images were pre-processed using SPM12 (Wellcome Department of Cognitive Neurology, Institute of Neurology, London, UK) running on Matlab 2018a (MathWorks Inc., Sherborn, MA). After an initial step of approximate manual re-orientation and positioning to MNI space, the spatial normalisation of each PET image into the MNI space was performed by spatial normalisation of the $\mathrm{CT}$ scan for each subject provided by the correction of attenuation, using the method and the template of the Clinical Toolbox for SPM (https:// www.nitrc.org/projects/clinicaltbx/). The voxel sizes of the written CT images were set to $1 \times 1 \times 1 \mathrm{~mm}^{3}$ for the digital controls and to $2 \times 2 \times 2 \mathrm{~mm}^{3}$ for the conventional controls. Each CT spatial normalisation procedure was subsequently applied to the respective PET images. Voxels of PET images recorded with the conventional 


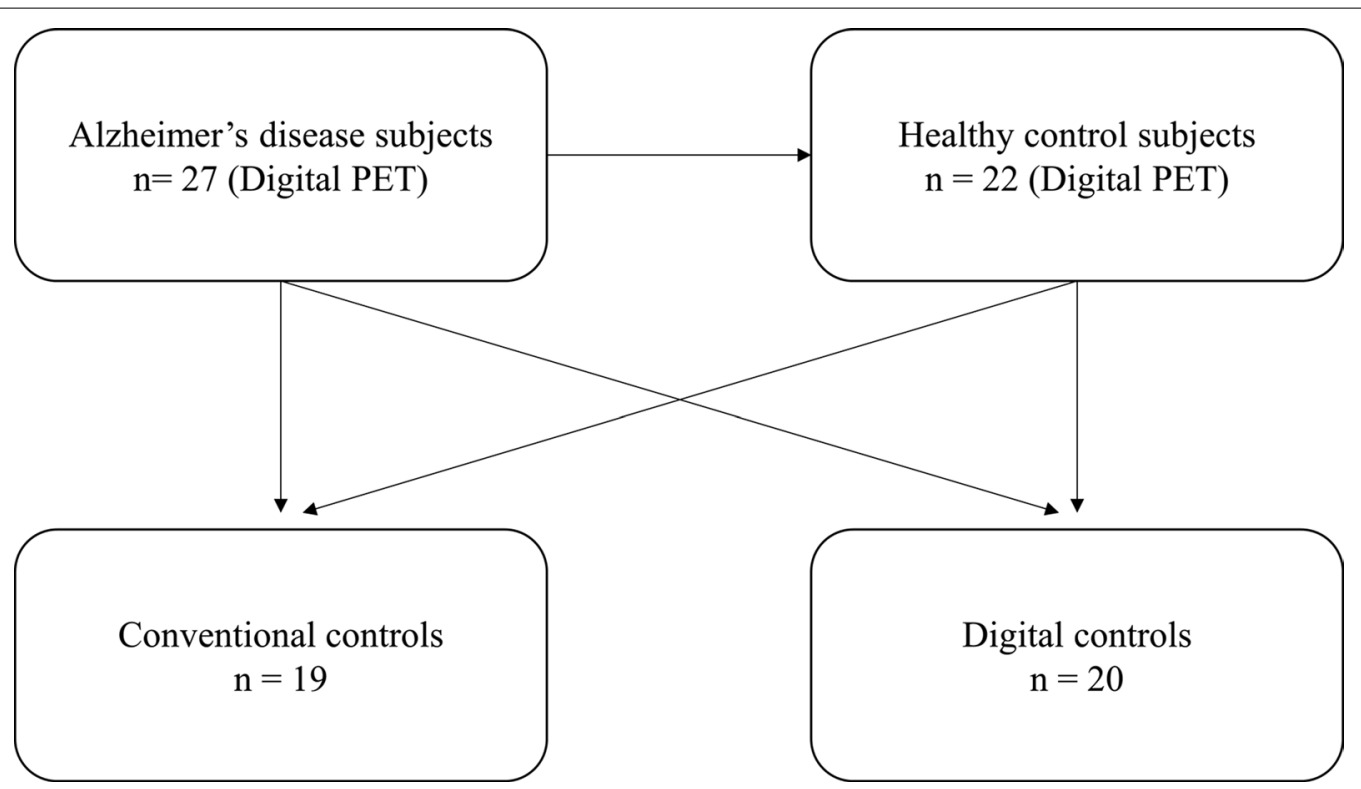

Fig. 1 Flowchart for the constitution of the two different groups of Alzheimer's disease and healthy control subjects, and the conventional and digital control databases. Black arrows are representative of analyses performed

camera during this step were therefore resampled from $2.7 \times 2.7 \times 2.7 \mathrm{~mm}^{3}$ to $2 \times 2 \times 2 \mathrm{~mm}^{3}$ whereas voxel sizes of the digital databases were not modified $(1 \times 1 \times 1$ $\mathrm{mm}^{3}$ ). To enable voxel-to-voxel analysis with these two control databases, $\mathrm{AD}$ and $\mathrm{HC}$ group datasets were normalised using the two voxel sizes. Partial volume effect corrections were applied to PET images using the MüllerGärtner (MG) method provided by the PETPVE12 toolbox [16]. White and grey matter segmentations needed for the 3-compartmental voxel-wise MG method were realised on CT scans using SPM segmentation tools, after careful visual analysis at the individual level to check the accuracy of this segmentation. The cerebellum was used as reference for the intensity normalisation of PET images because normalisations other than the proportional scaling have been proposed [17] and because the cerebellum is associated with a more accurate discrimination of patients with $\mathrm{AD}$ compared to controls [18]. All regions of interest (ROIs) used for intensity normalisation (all the cerebellum and vermis ROIs for the cerebellum, and all the pre- and post-central brain areas for the sensorimotor cortex) were extracted from the AAL atlas [19] after spatial normalisation to limit the interindividual anatomical heterogeneity. Finally, PET images were smoothed with an isotropic 3D Gaussian kernel of $12 \mathrm{~mm}$ FWHM to blur individual variations in gyral anatomy. Visual inspections of the images at the different stages of the pre-processing procedure ensured the quality and convergence of the different methods applied.
Semi-Quantitative Analyses (SQA) were performed at the group and individual level on a voxel-by-voxel basis using two-sample t-tests with an inclusive AD mask [20]. At the group level, AD and $\mathrm{HC}$ groups were compared with the conventional and digital controls using age and sex as covariates (clusters of decreased metabolic activity observed at $p<0.001$ for the voxel, cluster volume corrected by using the expected volume provided by SPM and based on the random field theory). We used exclusive masks to compare results obtained with SQA to conventional or digital controls. For $\mathrm{AD}$, an exclusive mask corresponding to the SPM-T map results of SQA to conventional controls was applied to the SQA of digital controls to highlight the additional clusters visualised with the digital system compared to the conventional system (and vice versa for the $\mathrm{HC}$ population).

At the individual level, each subject in the AD and $\mathrm{HC}$ group, was individually compared to the digital and conventional controls using a fully automated analysis as well as visual analyses (clusters of decreased metabolic activity observed at $p<0.005$ for the voxel, cluster volumes corrected to $\left.0.8 \mathrm{~cm}^{3}[6,21]\right)$. All clusters identified with SPM at the individual level were considered significant for the fully automated analysis.

The precise identification of each structure located by its MNI coordinates, its respective volume, and T-max intensity were extracted by using the report provided by the SPM xjView toolbox (http://www.alivelearn.net/xjvie $\mathrm{w})$. 


\section{Visual ratings at the individual level}

For the visual analyses, the SPM T-maps were projected onto three-dimensional rendering of T1-weighted MRI images using SPM surface rendering tool and onto 12 two-dimensional slices of T1-weighted MRI images using the Slice Display toolbox [22] (axial orientation, interslice spacing of $1 \mathrm{~cm}$ ). Representations were reviewed by three experienced observers (EM, EG and AV), who were blinded to the patient's clinical data. Raters were forced to give a dichotomous reading: Alzheimer's disease diagnosis or not pathological. A pattern of diffuse hypometabolic areas within the areas known to be involved in $\mathrm{AD}$ (mainly the bilateral posterior associative areas) was considered a positive scan. At the individual level, results were expressed as a consensual analysis for the positive diagnosis of AD.

\section{Statistical analysis}

Categorical variables are expressed as percentages and continuous variables as means and standard deviations. Due to the non-normality of variable distributions, Chi-2 and Kruskal-Wallis tests were performed for comparisons of categorical and continuous variables, respectively. For the comparisons of diagnostic performances at the individual level, Mc Nemar tests were used with corrections for multiple comparisons. A $p$ value $<0.05$ was considered as significant. All tests were performed with SPSS (SPSS Statistics for Windows, Version 20.0. Armonk, NY: IBM Corp).

\section{Results}

Population

As detailed in Table 1, no difference in age, sex and educational level were observed between the AD, HC groups, as well as the conventional and digital controls $(p>0.27)$.
As expected, AD patients showed lower levels of MMSE than $\mathrm{HC}$ subjects as well as conventional and digital controls $(p<0.01)$.

\section{At the group level}

In patients with $\mathrm{AD}, \mathrm{SQA}$ using digital controls enabled the detection of more marked hypometabolic areas $\left(+116 \mathrm{~cm}^{3}\right)$ when compared to SQA using conventional controls. There were 3 more clusters of significance identified with SQA using digital controls. The most extensive one involved the bilateral posterior associative areas, including the precuneus and the posterior cingulate (T-voxel max at 7.88). The two others, less extensive and with lower $\mathrm{T}$-voxel max values involved the bilateral anterior associative areas.

Details of significant additional hypometabolic regions obtained with SQA using digital controls in patients with $\mathrm{AD}$ are provided in Table 2 and Fig. 2.

Moreover, in healthy control subjects (HC), SQA using conventional controls showed more marked hypometabolic areas $\left(+17 \mathrm{~cm}^{3}\right)$ when compared to SQA using digital controls. There were 3 more significant clusters identified with SQA using conventional controls involving the precuneus, and the bilateral anterior associative areas. Details of significant hypometabolic areas obtained with SQA using conventional controls in healthy control subjects are provided in Table 3 and Fig. 3.

Of note, significant hypermetabolism was also shown with SQA using conventional controls both for comparisons to patients with $\mathrm{AD}\left(+21 \mathrm{~cm}^{3}\right)$ and to $\mathrm{HC}$ subjects $\left(+115 \mathrm{~cm}^{3}\right)$ whereas no significant hypermetabolism for these comparisons was observed for SQA using digital controls.

For further validation, the $\mathrm{AD}$ and $\mathrm{HC}$ groups, both acquired with the same digital PET scanner, were

Table 1 Characteristics of the Alzheimer's disease patients, healthy control subject's as well as the conventional and digital control groups

\begin{tabular}{|c|c|c|c|c|c|}
\hline Group & $\mathrm{AD}(n=27)$ & $\mathrm{HC}(n=22)$ & $\begin{array}{l}\text { Conventional controls } \\
(n=19)\end{array}$ & $\begin{array}{l}\text { Digital controls } \\
(n=20)\end{array}$ & $p$ values \\
\hline Age (years old) & $70.9 \pm 7.5$ & $67.7 \pm 10.4$ & $67.2 \pm 8.3$ & $66.6 \pm 8.5$ & 0.27 \\
\hline Sex (Female) & $14(52 \%)$ & $10(45 \%)$ & $8(42 \%)$ & $8(40 \%)$ & 0.86 \\
\hline Educational level & & & & & 0.60 \\
\hline None & 1 & 0 & 0 & 0 & \\
\hline Primary school & 11 & 9 & 14 & 12 & \\
\hline High school & 4 & 3 & 2 & 3 & \\
\hline College & 11 & 9 & 3 & 5 & \\
\hline NA & - & 1 & - & - & \\
\hline MMSE & $19.2 \pm 5.7^{*}$ & $28.7 \pm 0.9$ & $29.2 \pm 0.8$ & $29 \pm 0.9$ & $<0.01^{*}$ \\
\hline
\end{tabular}

$\mathrm{AD}$, Alzheimer's disease; $\mathrm{HC}$, healthy control; MMSE, mini-mental state examination score

${ }^{*} p<0.05$ for comparisons between groups 
Table 2 Additional AD patient clusters identified with SQA using digital controls as opposed to SQA using conventional controls (anatomical locations, spatial extent of significant clusters in $\mathrm{cm}^{3}$, MNI coordinates, maximal T-scores of the peak voxel) at a T-voxel threshold of 3.3, $\mathrm{k}$ cluster size $>1.86 \mathrm{~cm}^{3}$

\begin{tabular}{|c|c|c|c|c|c|}
\hline $\begin{array}{l}\text { Anatomical } \\
\text { location }\end{array}$ & Cluster size & $\mathbf{x}$ & $\mathbf{y}$ & $\mathbf{z}$ & T-score of peak \\
\hline \multicolumn{6}{|c|}{ Right and left cerebrum } \\
\hline $\begin{array}{l}\text { Cuneus } \\
\text { Precuneus } \\
\text { Posterior cingu- } \\
\quad \text { late } \\
\text { Superior parietal } \\
\text { Inferior parietal } \\
\text { Superior temporal } \\
\text { Middle temporal } \\
\text { Inferior temporal } \\
\text { Superior occipital } \\
\text { Middle occipital } \\
\text { Inferior occipital }\end{array}$ & 105 & -33 & -10 & -44 & 7.88 \\
\hline \multicolumn{6}{|l|}{ Right cerebrum } \\
\hline $\begin{array}{l}\text { Superior frontal } \\
\text { Middle frontal } \\
\text { Inferior frontal }\end{array}$ & 4 & 30 & 57 & -2 & 5.29 \\
\hline \multicolumn{6}{|l|}{ Left cerebrum } \\
\hline $\begin{array}{l}\text { Superior frontal } \\
\text { Middle frontal } \\
\text { Inferior frontal }\end{array}$ & 7 & -36 & 55 & 0 & 4.16 \\
\hline
\end{tabular}

compared at the group-level. The volume of cluster significance was similar to that observed in SQA using digital controls for patients with AD $\left(195 \mathrm{~cm}^{3}\right.$ with a T-voxel max value of significance at 7.48 , vs. $185 \mathrm{~cm}^{3}$ with a T-voxel max value of significance at 7.88 for the comparison between patients with $\mathrm{AD}$ and the digital controls).

\section{At the individual level}

The accuracy of SQA for discriminating AD using digital controls was higher than with conventional controls $(86$ vs. $80 \%$ ), with a higher sensitivity ( 89 vs. $78 \%$ ) and similar specificity ( $82 \%$ vs. $82 \%$ ) achieved with the fully automated analysis.

These results were confirmed by the visual analysis with an accuracy, sensitivity and specificity of respectively $84 \%, 85 \%, 82 \%$ for SQA using digital and $82 \%, 67 \%$, $100 \%$ for SQA using conventional controls.

Detailed diagnostic performances for both SQA using conventional and digital controls are reported in Table 4.

An illustration of SPM-T map images used for the visual analysis is displayed in Fig. 4. Figure 5 provides a galleria of individual patients with both SQA to conventional and digital controls.

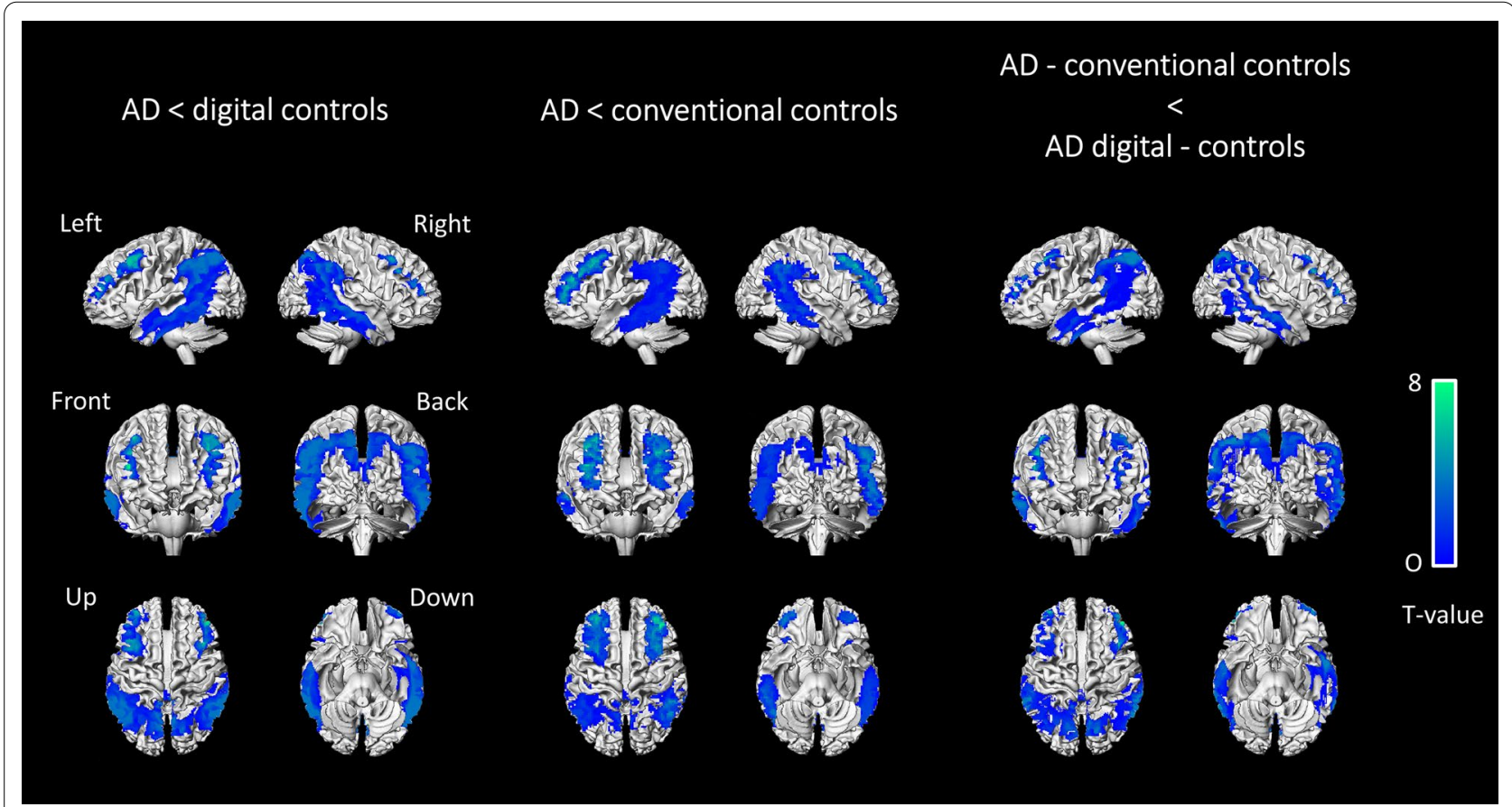

Fig. 2 Anatomical localisation of areas of decreased metabolic activity in AD patients $\left(p<0.001\right.$, uncorrected, $\left.k>1.86 \mathrm{~cm}^{3}\right)$ for SQA using digital controls (left panel), SQA using conventional controls (middle panel), and the significant additional hypometabolic areas obtained with SQA using digital controls compared to SQA using conventional controls (right panel), projected onto 3D volume rendering, spatially normalised and smoothed into the standard SPM template (T-voxel threshold value of 3.3) 
Table 3 Additional HC patient clusters identified with SQA using conventional controls as opposed to SQA using digital controls (anatomical locations, spatial extent of significant clusters in $\mathrm{cm}^{3}$, MNI coordinates, maximal T-scores of the peak voxel) at a T-voxel threshold of 3.3, $\mathrm{k}$ cluster size $>1.45 \mathrm{~cm}^{3}$

\begin{tabular}{llllll}
\hline Anatomical location & Cluster size & $\mathbf{x}$ & $\mathbf{y}$ & $\mathbf{z}$ & T-score of peak \\
\hline $\begin{array}{l}\text { Right cerebrum } \\
\begin{array}{l}\text { Middle frontal } \\
\text { Left cerebrum }\end{array}\end{array}$ & 5 & 34 & 2 & 38 & 5.73 \\
$\begin{array}{l}\text { Middle frontal } \\
\text { Right cerebrum } \\
\text { Precuneus }\end{array}$ & 7 & -24 & 2 & 46 & 5.01 \\
\hline
\end{tabular}

\section{Discussion}

This study aimed to assess whether the event of SQA and the evolution of digital PET imaging requires the control databases to evolve concomitantly. Diagnostic performances observed at the group and individual level show that the diagnostic accuracy of SQA on digital controls is improved compared to SQA on conventional controls, particularly as it relates to the detection sensitivity of AD. This observation is an argument which supports the development of digital control databases for SQA of brain ${ }^{18}$ F-FDG PET images for clinical practice.

Digital PET technology is associated with improvements in image quality, specifically better spatial resolution and signal-to-noise ratios compared to conventional PET cameras [2]. These distinct image qualities lead to problematic head-to-head comparisons between digital and conventional PET images as reflected in our study by the relatively poor diagnostic performance obtained for SQA using conventional controls for discriminating AD. However, all currently implemented control databases in dedicated software for automated SQA in clinical practice still rely on conventional PET control images $[9,13]$. Of course, it is now possible to implement local databases in the majority of these types of software, but establishing control databases acquired with digital PET technology remains an extensive undertaking, particularly because it involves a relative recently implemented technology.

Our present study shows that implementing a control database acquired with digital PET technology yields an increase in the detection sensitivity of AD patients, not only at the group level $\left(+116 \mathrm{~cm}^{3}\right.$ of detected hypometabolism volume, Table 2) but also at the individual

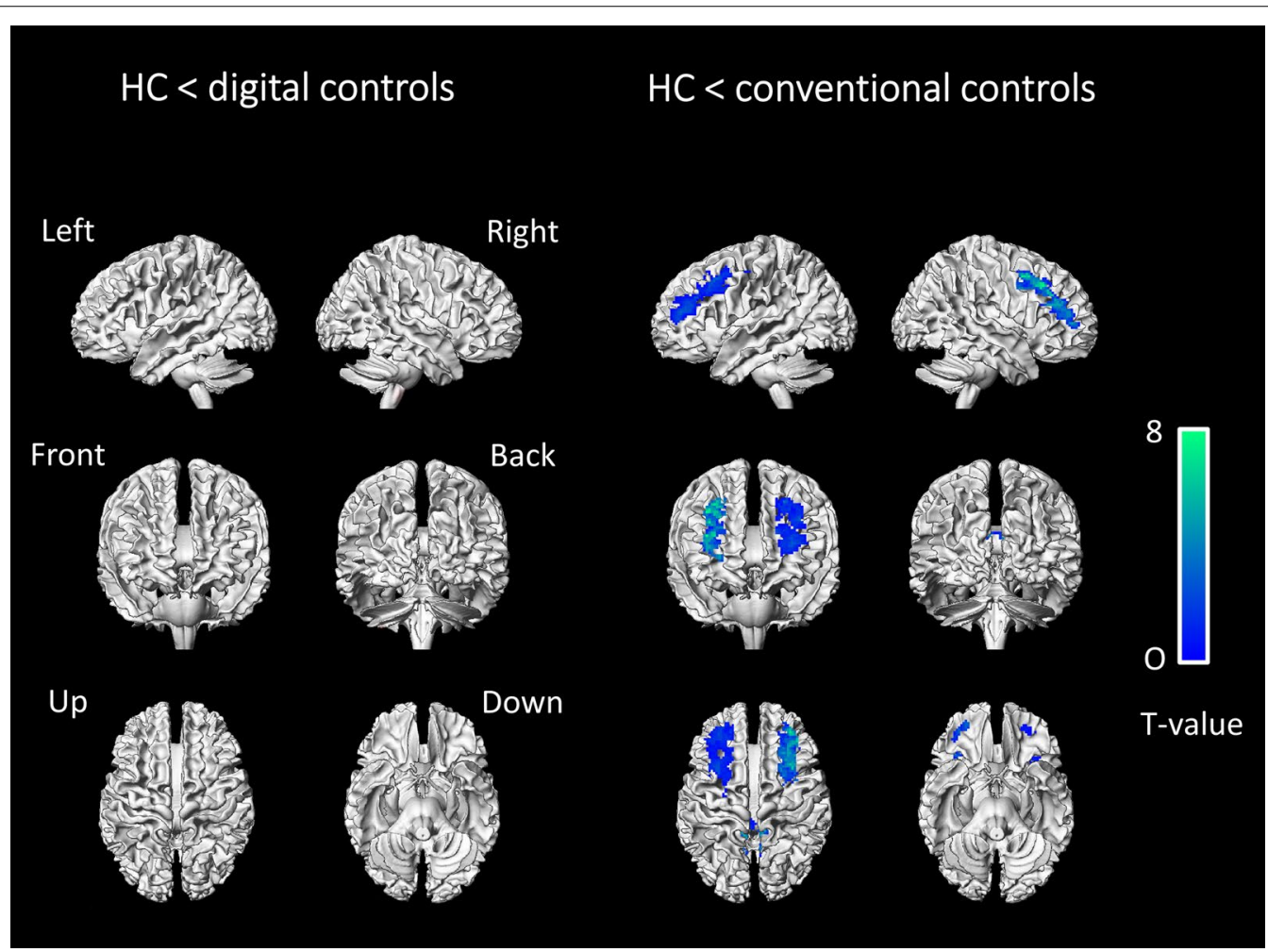

Fig. 3 Anatomical localisation of areas of decreased metabolic activity in $\mathrm{HC}$ subjects $\left(p<0.001\right.$, uncorrected, $\left.k>1.45 \mathrm{~cm}^{3}\right)$, with SQA using digital controls (left panel) and SQA using conventional controls (right panel), projected onto 3D volume rendering, spatially normalised and smoothed into the standard SPM template (T-voxel threshold value of 3.3) 
Table 4 Diagnostic performances of SQA using conventional and digital controls at the individual level with fully automated and visual analyses

\begin{tabular}{|c|c|c|c|c|}
\hline & Accuracy (\%) & Sensitivity (\%) & Specificity (\%) & $p$ value \\
\hline Fully automated & & & & $<0.01^{*}$ \\
\hline SQA using conventional controls & 80 & 78 & 82 & \\
\hline SQA using digital controls & 86 & 89 & 82 & \\
\hline Visual & & & & $=0.01^{*}$ \\
\hline SQA using conventional controls & 82 & 67 & 100 & \\
\hline SQA using digital controls & 84 & 85 & 82 & \\
\hline
\end{tabular}

* For the comparison between SQA using conventional and digital controls

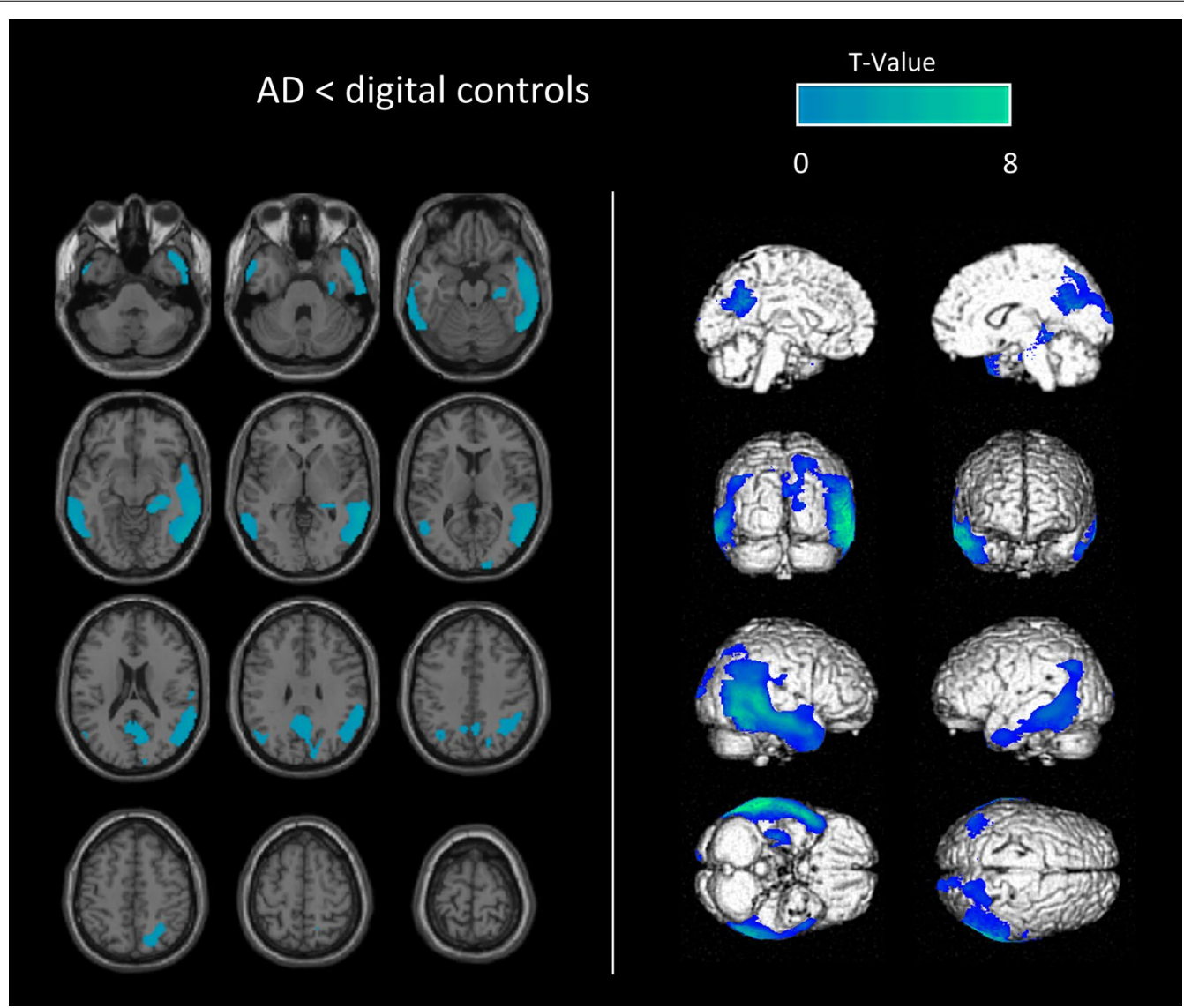

Fig. 4 Example of SPM-T map images used for the visual analysis (semi-quantitative analysis to digital controls) of a patient with AD (79-year-old man with MMSE score of 10). SPM-T maps are projected onto two-dimensional slices of T1-weighted MRI (from the base to the top of the skull, left panel) and 3D-rendered volumes (right panel)

level (sensitivity of detection increased from 78 to $89 \%$, Table 4). A high detection sensitivity is primordial in the diagnosis of AD since ${ }^{18} \mathrm{~F}$-FDG PET is a biomarker of neurodegeneration, which contributes to the ATN classification, the $\mathrm{N}$ biomarker being directly associated with cognitive impairment in patients suspected to have neurodegenerative diseases [14].
All results in the current study were initially obtained using a fully automated analysis, which supports the objective nature of our observations in both the group and individual level analyses. This original fully automated methodology, which necessitated an adaptation of the levels of significance to detect anomalies, was exclusively based on the SPM software. From a clinical 


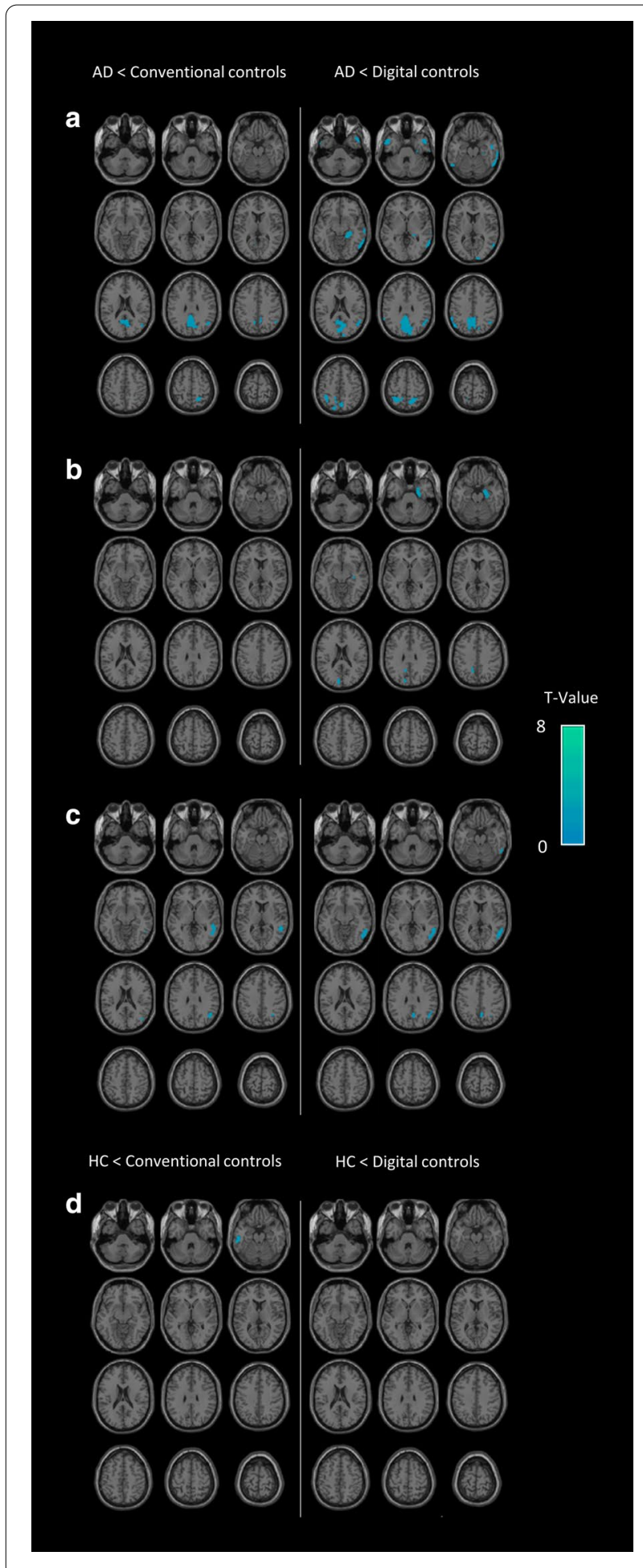

standpoint and at the individual level, this fully automated analysis was nevertheless consistent with the visual analysis, using a methodology that is very similar to that applied in previously published SQA studies $[6,12]$. By using this visual analysis, the diagnostic
Fig. 5 Galleria of individual examples with both semi-quantitative analyses to conventional and digital controls. Three examples of SPM-T map images from semi-quantitative analyses to conventional (left panel) and digital (right panel) controls of patients with AD (a 74-year-old woman with MMSE score of 19, b 70-year-old woman with MMSE score of 21, c 66-year-old man with MMSE score of 23). One example of SPM-T map images from semi-quantitative analyses to conventional (left panel) and digital (right panel) controls from a healthy subject (d 54-year-old woman with MMSE score of 29). SPM-T maps are projected onto two-dimensional slices of T1-weighted MRIs (from the base to the top of the skull)

performance of SQA for discriminating AD with the digital controls observed in our study (accuracy, sensitivity and specificity of respectively 84,85 and $82 \%$ ) was within the range of previously reported results (70-97.5\% for accuracy, $62.3-96 \%$ for sensitivity and $84-99 \%$ for specificity) [6, 8-12, 23-25].

SQA at the individual level was also performed using an intensity normalisation based on the sensorimotor cortex, which has been suggested to improve diagnostic performance of SQA [26]. The finds results were comparable to intensity normalisation on cerebellum. SQA on digital controls show respectively accuracy, sensitivity and specificity $84 \%, 89 \%$ and $77 \%$ (vs. $86 \%, 89 \%$ and $82 \%)$. SQA on conventional controls show respectively accuracy, sensitivity and specificity $73 \%, 52 \%$ and $100 \%$ (vs. $80 \% 78 \%$ and $82 \%$ ).

The post-filter used (Gaussian kernel of $12 \mathrm{~mm}$ FWHM) can be decreased relative to the size of the voxels used particularly for digital PET scans. However, when smoothing images with a Gaussian kernel of $4 \mathrm{~mm}$ FWHM, the accuracy to detect AD, respectively versus digital and conventional controls, remained unchanged ( $86 \%$ vs. $86 \%$ and $82 \%$ vs. $80 \%$ ).

The main limitation of our study results from the fact that controls included in the conventional and digital control databases were different individuals. From an ethical perspective, it remains problematic to establish control databases acquired in parallel with both the conventional and digital PET systems, even if it would be feasible to scan the same set of HC subjects within a few months on two different scanners using the half-dose permitted by the high sensitivity of the digital PET. It should however be noted that controls included in our conventional and digital databases did not exhibit any differences in age, sex, MMSE and educational level when compared to each other, or when compared to the $\mathrm{AD}$ and $\mathrm{HC}$ groups. In addition, these two, distinct conventional and digital control databases are representative samples from current daily clinical practice. The main objective of the current study was to assess whether there is indeed a requirement to establish digital control databases when acquisitions are 
performed with the new digital PET system. A secondary issue that may be addressed is that the sample size of conventional and digital control databases is rather small ( $n=19$ and 20). This number of controls is nevertheless known to be sufficient to accurately perform group analyses with SPM [27].

Overall, in light of recent digital PET technology developments and considering that SQA is now clearly recommended for brain ${ }^{18} \mathrm{~F}$-FDG PET image analysis, there is an urgent need to establish digital PET control databases for SQA of brain ${ }^{18} \mathrm{~F}$-FDG PET images. This would be particularly helpful for improving the sensitivity required to detect AD patients. Large healthy control databases should be constituted and shared through the multicentre community using standardised imaging protocols.

\section{Acknowledgements}

The authors thank the Nancyclotep Imaging Platform for their support.

\section{Authors' contributions}

All authors contributed significantly to the analysis and interpretation of the data (EM, MD, TJ, CM), to the writing of the manuscript (EM, MD, AV) and to the revision of the manuscript (TJ, CM, EG, AV). All authors read and approved the final manuscript.

\section{Funding}

None.

\section{Availability of data and materials}

The data that support the findings of this study are available on request from the corresponding author (AV).

\section{Code availability}

Not applicable.

\section{Ethics approval and consent to participate}

All procedures performed in this study involving human participants were in accordance with the ethical standards of the institutional and/or national research committees and with the 1964 Helsinki declaration and its latest amendments or comparable ethics standards. Subjects included in the two control databases were derived from prospective studies (NCT02858167 and NCT03345290).

\section{Consent for publication}

Not applicable.

\section{Competing interests}

The authors disclose no potential conflicts of interest related to the present work.

\section{Informed consent}

Informed consent was obtained for each subject included in the two retrospectively selected groups. This study was approved on January 16, 2020 by the local ethics committee (NCT04163276, Study ID Numbers: 2019PI238).

\footnotetext{
Author details

1 Department of Nuclear Medicine and Nancyclotep Imaging Platform, CHRU Nancy, Université de Lorraine, Rue du Morvan, 54500 Vandoeuvre-les-Nancy, France. ${ }^{2}$ IADI, INSERM U1254, Université de Lorraine, 54000 Nancy, France. ${ }^{3}$ Clinical Memory and Research Center, Department of Geriatrics, CHRU Nancy, Université de Lorraine, 2LPN EA 7489, 54000 Nancy, France. ${ }^{4}$ Department of Biochemistry, Molecular Biology and Nutrition CHRU Nancy, Université de Lorraine, 54000 Nancy, France. ${ }^{5}$ APHM, CNRS, Centrale Marseille, Institut Fresnel, Timone Hospital, CERIMED, Nuclear Medicine Department, Aix Marseille Univ, Marseille, France.
}

Received: 31 July 2020 Accepted: 23 November 2020

Published online: 30 November 2020

\section{References}

1. Salvadori J, Odille F, Verger A, Olivier P, Karcher G, Marie P-Y, et al. Headto-head comparison between digital and analog PET of human and phantom images when optimized for maximizing the signal-to-noise ratio from small lesions. EJNMMI Phys. 2020;7(1):11.

2. Salvadori J, Imbert L, Perrin M, Karcher G, Lamiral Z, Marie P-Y, et al. Headto-head comparison of image quality between brain 18F-FDG images recorded with a fully digital versus a last-generation analog PET camera. EJNMMI Res. 2019;9(1):61.

3. Nobili F, Arbizu J, Bouwman F, Drzezga A, Agosta F, Nestor P, et al. European Association of Nuclear Medicine and European Academy of Neurology recommendations for the use of brain $18 \mathrm{~F}$-fluorodeoxyglucose positron emission tomography in neurodegenerative cognitive impairment and dementia: Delphi consensus. Eur J Neurol. 2018;25(10):1201-17.

4. Arbizu J, Festari C, Altomare D, Walker Z, Bouwman F, Rivolta J, et al. Clinical utility of FDG-PET for the clinical diagnosis in MCI. Eur J Nucl Med Mol Imaging. 2018;45(9):1497-508.

5. Nestor PJ, Altomare D, Festari C, Drzezga A, Rivolta J, Walker Z, et al. Clinical utility of FDG-PET for the differential diagnosis among the main forms of dementia. Eur J Nucl Med Mol Imaging. 2018;45(9):1509-25.

6. Perani D, Della Rosa PA, Cerami C, Gallivanone F, Fallanca F, Vanoli EG, et al. Validation of an optimized SPM procedure for FDG-PET in dementia diagnosis in a clinical setting. Neurolmage Clin. 2014;6:445-54.

7. Van Der Gucht A, Verger A, Guedj E, Malandain G, Hossu G, Yagdigul $Y$, et al. Age-related changes in FDG brain uptake are more accurately assessed when applying an adaptive template to the SPM method of voxel-based quantitative analysis. Ann Nucl Med. 2015;29(10):921-8.

8. Nobili F, Festari C, Altomare D, Agosta F, Orini S, Van Laere K, et al. Automated assessment of FDG-PET for differential diagnosis in patients with neurodegenerative disorders. Eur J Nucl Med Mol Imaging. 2018;45(9):1557-66

9. Ishii K, Kono AK, Sasaki H, Miyamoto N, Fukuda T, Sakamoto S, et al. Fully automatic diagnostic system for early- and late-onset mild Alzheimer's disease using FDG PET and 3D-SSP. Eur J Nucl Med Mol Imaging. 2006;33(5):575-83.

10. Burdette JH, Minoshima S, Vander Borght T, Tran DD, Kuhl DE. Alzheimer disease: improved visual interpretation of PET images by using three-dimensional stereotaxic surface projections. Radiology. 1996;198(3):837-43.

11. Kono AK, Ishii K, Sofue K, Miyamoto N, Sakamoto S, Mori E. Fully automatic differential diagnosis system for dementia with Lewy bodies and Alzheimer's disease using FDG-PET and 3D-SSP. Eur J Nucl Med Mol Imaging. 2007;34(9):1490-7.

12. Lehman VT, Carter RE, Claassen DO, Murphy RC, Lowe V, Petersen RC, et al. Visual assessment versus quantitative three-dimensional stereotactic surface projection fluorodeoxyglucose positron emission tomography for detection of mild cognitive impairment and Alzheimer disease. Clin Nucl Med. 2012;37(8):721-6.

13. Sun X, Liu F, Liu Q, Gai Y, Ruan W, Wimalarathne DN, et al. Quantitative research of 11C-CFT and 18F-FDG PET in Parkinson's disease: a pilot study with NeuroQ software. Front Neurosci. 2019;13:299.

14. Jack CR, Bennett DA, Blennow K, Carrillo MC, Dunn B, Haeberlein SB, et al. NIA-AA research framework: toward a biological definition of Alzheimer's disease. Alzheimers Dement J Alzheimers Assoc. 2018;14(4):535-62.

15. Manca C, Rivasseau Jonveaux T, Roch V, Marie P-Y, Karcher G, Lamiral Z, et al. Amyloid PETs are commonly negative in suspected Alzheimer's disease with an increase in CSF phosphorylated-tau protein concentration but an $A \beta 42$ concentration in the very high range: a prospective study. J Neurol. 2019;266(7):1685-92.

16. Gonzalez-Escamilla G, Lange C, Teipel S, Buchert R, Grothe MJ, Alzheimer's Disease Neuroimaging Initiative. PETPVE12: an SPM toolbox for partial volume effects correction in brain PET_-application to amyloid imaging with AV45-PET. Neurolmage. 2017;147:669-77. 
17. Minoshima S, Frey KA, Foster NL, Kuhl DE. Preserved pontine glucose metabolism in Alzheimer disease: a reference region for functional brain image (PET) analysis. J Comput Assist Tomogr. 1995;19(4):541-7.

18. Dukart J, Mueller K, Horstmann A, Vogt B, Frisch S, Barthel H, et al. Differential effects of global and cerebellar normalization on detection and differentiation of dementia in FDG-PET studies. Neurolmage. 2010;49(2):1490-5.

19. Tzourio-Mazoyer N, Landeau B, Papathanassiou D, Crivello F, Etard O, Delcroix N, et al. Automated anatomical labeling of activations in SPM using a macroscopic anatomical parcellation of the MNI MRI single-subject brain. Neurolmage. 2002;15(1):273-89.

20. Herholz K, Salmon E, Perani D, Baron JC, HolthoffV, Frölich L, et al. Discrimination between Alzheimer dementia and controls by automated analysis of multicenter FDG PET. Neurolmage. 2002;17(1):302-16.

21. Della Rosa PA, Cerami C, Gallivanone F, Prestia A, Caroli A, Castiglioni I, et al. A standardized [18F]-FDG-PET template for spatial normalization in statistical parametric mapping of dementia. Neuroinformatics. 2014;12(4):575-93.

22. Zandbelt B. bramzandbelt/slice_display [Internet]. 2019 [cité 13 mai 2020]. Disponible sur: https://github.com/bramzandbelt/slice_display

23. Morbelli S, Brugnolo A, Bossert I, Buschiazzo A, Frisoni GB, Galluzzi S, et al. Visual versus semi-quantitative analysis of 18F-FDG-PET in amnestic $\mathrm{MCl}$ : an European Alzheimer's Disease Consortium (EADC) project. J Alzheimers Dis. 2015;44(3):815-26.
24. Rabinovici GD, Rosen HJ, Alkalay A, Kornak J, Furst AJ, Agarwal N, et al. Amyloid vs FDG-PET in the differential diagnosis of AD and FTLD. Neurology. 2011;77(23):2034-42

25. Ng S, Villemagne VL, Berlangieri S, Lee S-T, Cherk M, Gong SJ, et al. Visual assessment versus quantitative assessment of 11C-PIB PET and 18F-FDG PET for detection of Alzheimer's disease. J Nucl Med Off Publ Soc Nucl Med. 2007;48(4):547-52.

26. Yakushev I, Landvogt C, Buchholz H-G, Fellgiebel A, Hammers A, Scheurich $A$, et al. Choice of reference area in studies of Alzheimer's disease using positron emission tomography with fluorodeoxyglucose-F18. Psychiatry Res. 2008;164(2):143-53.

27. Signorini M, Paulesu E, Friston K, Perani D, Colleluori A, Lucignani G, et al. Rapid assessment of regional cerebral metabolic abnormalities in single subjects with quantitative and nonquantitative [18F]FDG PET: a clinical validation of statistical parametric mapping. Neurolmage. 1999;9(1):63-80.

\section{Publisher's Note}

Springer Nature remains neutral with regard to jurisdictional claims in published maps and institutional affiliations.

\section{Submit your manuscript to a SpringerOpen ${ }^{\circ}$ journal and benefit from:}

- Convenient online submission

- Rigorous peer review

- Open access: articles freely available online

- High visibility within the field

- Retaining the copyright to your article

Submit your next manuscript at $\boldsymbol{\nabla}$ springeropen.com 